\title{
SPH modelling of the interaction between free-surface flows of a viscoplastic fluid and a structure
}

\author{
M. Labbé \& D. Laigle \\ Irstea, UR ETGR, Saint-Martin-d'Héres, France
}

\begin{abstract}
Debris flows are rapid, unsteady gravity-driven flows which can cause vast amounts of damage to infrastructures and endanger populations. It is therefore crucial to be able to properly model the behaviour of these flows, to better predict their impact. The aim of this study is to simulate these flows, determine how the presence of an obstacle in the path of the flow influences the propagation of the fluid, and to measure the pressures exerted by the flow on the obstacle, for various flow regimes.

In this paper, we present a 2-D Smooth Particle Hydrodynamics (SPH) model capable of simulating a viscoplastic fluid. The Herschel-Bulkley model, which can accurately describe the behaviour of a muddy debris flow, is introduced in the SPH equations of Ferrari et al. (A new 3D parallel SPH scheme for free surface flows. Computers \& Fluids, 38(6), pp. 1203-1217, 2009). The resulting code is validated by simulating a steady flow. It is then used to simulate a Herschel-Bulkley fluid propagating from a tank onto an incline and past a rectangular obstacle. The simulations confirm the existence of two regimes observed experimentally: a deadzone regime for small values of the Froude number and a jet regime for large values of the Froude number. Results show that the pressure exerted on the obstacle can be described by a combination of a gravitational component and a kinetic component, the latter taking over as the flow transitions from the dead-zone regime to the jet regime.

Keywords: debris flows, SPH, viscoplastic fluid, numerical simulation.
\end{abstract}




\section{Introduction}

Mountain areas are subjected to intense erosive processes. These processes can yield large-scale gravity-driven flows of geomaterials: rock falls, landslides, avalanches and debris flows, among others.

Debris flows in particular are rapid, unsteady gravity-driven flows constituted of a matrix of water and fine sediment (clay, silt and sand) capable of carrying debris of various sizes, including large boulders. When their fine fraction is large enough, their behaviour is that of a shear-thinning viscoplastic fluid [1].

They can cause damage to infrastructures and property as well as endanger populations [4]. It is therefore important to quantify the impact these flows can have on structures. This paper focuses on the application of a 2-D SPH method to the simulation of a free-surface flow of a Herschel-Bulkley fluid impacting a rectangular obstacle. We observe the influence of the obstacle on the flow at various regimes, and we study the pressure exerted by the flow on the obstacle.

\section{The SPH method and its implementation}

\subsection{Smooth Particle Hydrodynamics}

The SPH method is a mesh-free numerical method initially introduced by Gingold and Monaghan [5] and Lucy [6] to study astrophysical problems. It has since found many applications in fluid dynamics [7]. Because of its mesh-free nature, it is particularly well-suited for the simulation of the propagation of free-surface flows such as debris flows $[8,9]$.

The classical Navier-Stokes equations for a fluid are:

$$
\begin{aligned}
\frac{d \rho}{d t} & =-\rho \operatorname{div} \vec{u} \\
\frac{D \vec{u}}{D t} & =\frac{1}{\rho} \operatorname{div} \sigma+\vec{g}=-\frac{1}{\rho} \vec{\nabla} P+\frac{1}{\rho} \operatorname{div} \tau+\vec{g}
\end{aligned}
$$

where $\rho$ is the density of the fluid, $\vec{u}$ the local velocity of the fluid and $D \vec{u} / D t$ its material derivative, $\sigma$ is the total stress tensor, $\tau$ is the deviatoric stress tensor, $P$ is the local pressure, and $\vec{g}$ is the gravity.

$\mathrm{SPH}$ consists in discretizing the continuous medium into particles that represent small fluid elements, moving with the material velocity and carrying physical properties such as density, pressure and stresses. SPH relies on an interpolation to approximate the value of a function $f$ and its spatial derivatives at any point $\vec{x}$ :

$$
\begin{aligned}
f(\vec{x}) & =\sum_{j=1}^{N} \frac{m_{j}}{\rho_{j}} f\left(\vec{x}_{j}\right) W\left(\vec{x}-\vec{x}_{j}\right) \\
\vec{\nabla} f(\vec{x}) & =\sum_{j=1}^{N} \frac{m_{j}}{\rho_{j}} f\left(\vec{x}_{j}\right) \vec{\nabla} W\left(\vec{x}-\vec{x}_{j}\right)
\end{aligned}
$$


where $N$ is the number of SPH particles, and $m_{j}, \rho_{j}, \vec{x}_{j}$ are the mass, density and position of particle $j . W$ is the smoothing kernel function. Here, we use a cubic spline [10].

The SPH code used in this study solves eqns (1-2) in two dimensions. A flux term, introduced by Ferrari et al. [2], is added to the classical SPH massconservation equation to stabilize the pressure field, yielding the following equations:

$$
\begin{aligned}
\frac{d \rho_{i}}{d t} & =\sum_{j=1}^{N} m_{j}\left[\left(\vec{v}_{i}-\vec{v}_{j}\right) \cdot \vec{\nabla}_{i} W_{i j}+\vec{n}_{i j} \cdot \vec{\nabla}_{i} W_{i j}\left(\frac{c}{\rho_{j}}\left(\rho_{i}-\rho_{j}\right)\right)\right] \\
\frac{d v_{i}^{\alpha}}{d t} & =\sum_{j=1}^{N} \sum_{\beta} m_{j}\left(\frac{\sigma_{i}^{\alpha \beta}}{\rho_{i}^{2}}+\frac{\sigma_{j}^{\alpha \beta}}{\rho_{j}^{2}}\right) \frac{\partial W_{i j}}{\partial x_{i}^{\beta}}+g_{\alpha} \\
& =\sum_{j=1}^{N}\left[-m_{j}\left(\frac{P_{i}}{\rho_{i}^{2}}+\frac{P_{j}}{\rho_{j}^{2}}\right) \frac{\partial W_{i j}}{\partial x_{i}^{\alpha}}+\sum_{\beta} m_{j}\left(\frac{\tau_{i}^{\alpha \beta}}{\rho_{i}^{2}}+\frac{\tau_{j}^{\alpha \beta}}{\rho_{j}^{2}}\right) \frac{\partial W_{i j}}{\partial x_{i}^{\beta}}\right]+g_{\alpha},
\end{aligned}
$$

where $\vec{n}_{i j}$ is a unitary vector pointing from particle $i$ to particle $j, c$ is the speed of sound, and indices $\alpha$ and $\beta$ refer to Cartesian coordinates.

The pressure $P$ is computed from the density using the following equation of state:

$$
P=c^{2}\left(\rho-\rho_{0}\right),
$$

where $\rho_{0}$ is a reference density. The value of $c$ is chosen so that the artificial compressibility is negligible. It is constant in our simulations.

\subsection{Herschel-Bulkley model}

The rheology of muddy debris flows can be described by the Herschel-Bulkley model [1], in which the norm of the deviatoric stress tensor $\tau$ is given by the following equation:

$$
\begin{cases}|\tau|=\tau_{c}+K \dot{\gamma}^{n} & \text { if } \dot{\gamma} \neq 0 \\ |\tau| \leq \tau_{c} & \text { otherwise }\end{cases}
$$

where $\tau_{c}$ is the yield stress, $K$ is the consistency and $n$ the power-law index of the Herschel-Bulkley fluid. $\dot{\gamma}=\sqrt{2 \operatorname{tr} \dot{\varepsilon}^{2}}$ is the shear rate, where $\dot{\varepsilon}$ is the strain rate tensor.

To simulate a Herschel-Bulkley fluid, we introduce an apparent dynamic viscosity in our model:

$$
\eta_{a p p}=\frac{\tau_{c}}{\dot{\gamma}}+K \dot{\gamma}^{n-1} .
$$

The SPH formulation of the strain rate tensor $\dot{\varepsilon}$, on which $\dot{\gamma}$ depends, is:

$$
\dot{\varepsilon}_{i}^{\alpha \beta}=-\frac{1}{2} \sum_{j=1}^{N} \frac{m_{j}}{\rho_{i}}\left(u_{i j}^{\alpha} \frac{\partial W_{i j}}{\partial x^{\beta}}+u_{i j}^{\beta} \frac{\partial W_{i j}}{\partial x^{\alpha}}\right)
$$


and the deviatoric stress is then $\tau=2 \eta_{a p p} \dot{\varepsilon}$.

However, as $\eta_{a p p}$ goes to infinity when $\dot{\gamma}$ goes to zero, we introduce a maximum viscosity $\eta_{\max }$ :

$$
\tau=2 \min \left(\eta_{\text {app }}, \eta_{\max }\right) \dot{\varepsilon},
$$

meaning that for small values of $\dot{\gamma}$, the fluid behaves like a highly viscous Newtonian fluid.

\subsection{Validation on steady flows}

To validate our numerical model, we simulated a steady uniform free-surface flow down a slope, for which an analytical solution exists [11]. For such flows of Herschel-Bulkley fluids, a non-sheared zone (plug) forms above a sheared zone of thickness:

$$
h_{s}=h-\frac{\tau_{c}}{\rho g \sin \theta},
$$

where $h$ is the total height of the fluid and $\theta$ is the slope angle.

The velocity of the plug is given by:

$$
u_{p l u g}=\frac{n}{n+1}\left(\frac{\rho g h_{s}^{n+1} \sin \theta}{K}\right)^{1 / n} .
$$

The velocity profile in the sheared layer is given by:

$$
u_{x}(z)=u_{\text {plug }}\left[1-\left(1-\frac{z}{h_{s}}\right)^{\frac{n+1}{n}}\right], \quad 0 \leq z \leq h_{s} .
$$

Figure 1 shows the numerical results obtained for a slope $\theta=8^{\circ}$ along with the analytical solution. The agreement between the two profiles indicates that the code is able to reproduce a steady free-surface flow of Herschel-Bulkley fluid very accurately, and should be able to simulate the interaction between an unsteady flow and a structure.

\section{Numerical experiments and observations}

\subsection{Numerical setup}

Figure 2 shows the numerical setup used in our simulations. It is inspired by experiments conducted in a flume at our laboratory: fluid is released from a tank by an opening at the bottom, onto an inclined plane. A rectangular obstacle is placed in the path of the fluid, and the pressure exerted by the fluid on the obstacle is recorded with a numerical sensor located in the middle of the wall facing the incoming flow. The level of fluid in the tank is kept constant by inserting new particles regularly, to maintain a steady regime behind the front of the flow.

Multiple runs were conducted, for slope angles varying between $\theta=3^{\circ}$ and $12^{\circ}$. For each angle, we first ran a simulation to determine the height of the fluid $h$ in 


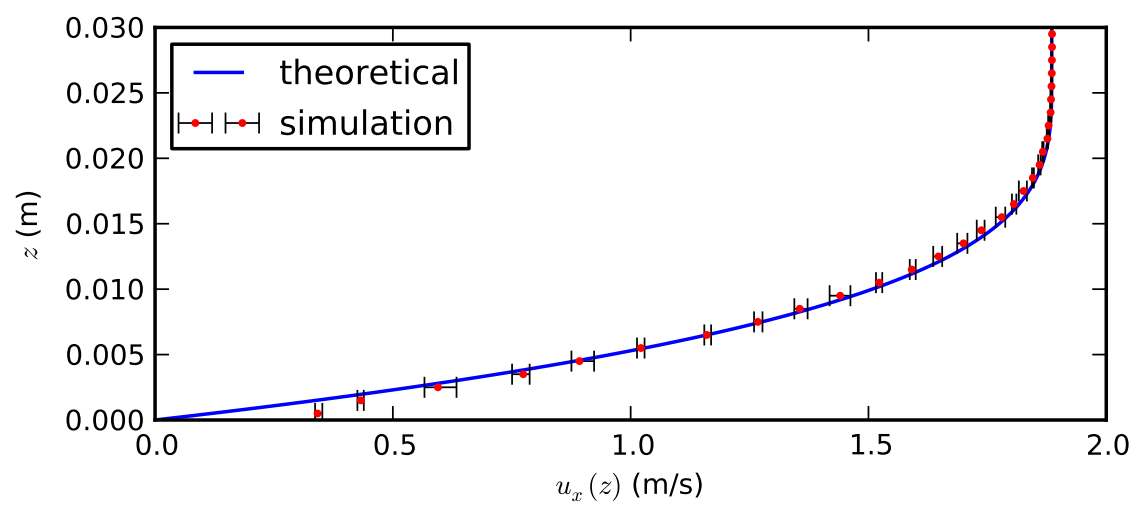

Figure 1: Theoretical velocity profile (blue line) and numerical results (red dots) for a free-surface Poiseuille flow of Herschel-Bulkley fluid. The error bars indicate the variability between profiles taken at different positions along the $x$-axis.

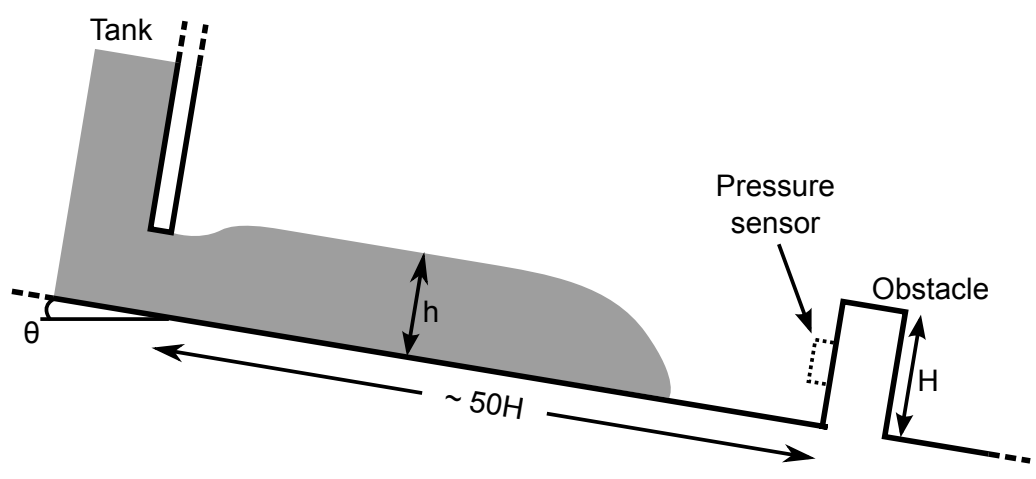

Figure 2: Numerical setup used in the simulations.

the absence of the obstacle, and the average front velocity $u$. From these data we can calculate the Froude number $F r=u / \sqrt{g h \cos \theta}$ of the flow. The range of angles studied here corresponds to values of $F r$ ranging from 0.52 to 2.22.

A second simulation was then run with an obstacle in the path of the flow. The height of the obstacle $H$ was chosen so that the ratio $H / h=1.16$ is the same for all slope angles (in accordance with previous lab experiments). For each slope angle, we observed the evolution of the free surface and the internal properties of the flow. We also studied the evolution of the pressure exerted by the fluid on the obstacle. 

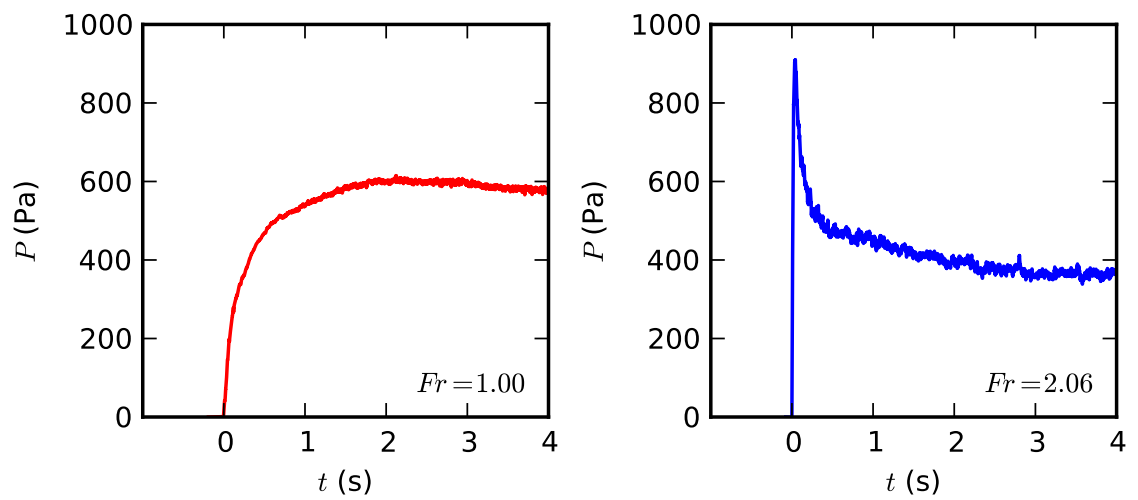

Figure 3: Time evolution of the pressure on the obstacle in the dead-zone regime $(F r=1.00$, left $)$ and the jet regime $(F r=2.06$, right $)$.

\subsection{Flow regimes}

Figure 3 shows the evolution of the pressure measured at the center of the obstacle as a function of time, for $F r=1.00$ and $F r=2.06$.

Two regimes can be observed. For small values of the Froude number $(\mathrm{Fr}<$ 1.5), the fluid rises smoothly along the obstacle, and a large zone of motionless fluid (a dead zone) forms at the foot of the wall. The pressure measured on the obstacle rises smoothly and reaches a maximum after a few seconds. We call this regime the dead-zone regime.

For $\mathrm{Fr}>1.5$, a vertical jet forms when the fluid impacts the obstacle. The pressure peaks to its maximum value in a few tenths of a second, then decreases as a small dead zone forms. We name this the jet regime.

\subsection{Zone of influence}

The presence of a dead zone alters the shape of the free surface of the fluid located upstream of the obstacle. The region in which the free surface is modified is called the zone of influence. Figure 4 shows the length of the zone of influence divided by the obstacle height $L_{Z I} / H$ as a function of $F r$. In the jet regime, $L_{Z I}$ is small (less than $5 H$ ) and does not vary greatly with $F r$. For $F r<1.5, L_{Z I}$ grows rapidly from $5 \mathrm{H}$ to $25 \mathrm{H}$ as the slope angle (and therefore $\mathrm{Fr}$ ) decreases.

\subsection{Pressure exerted on the obstacle}

For each slope angle, we have recorded the evolution of the pressure on the obstacle with a numerical sensor in the middle of the upstream wall. The maximum values attained during each simulation are shown as a function of $\mathrm{Fr}$ on figure 5. 


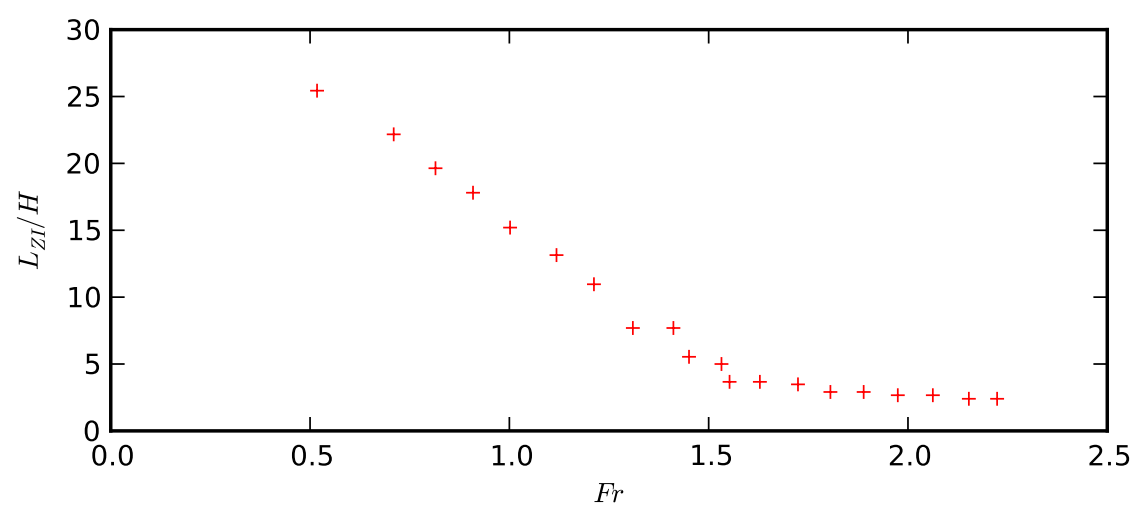

Figure 4: Length of the zone of influence divided by the height of the obstacle.

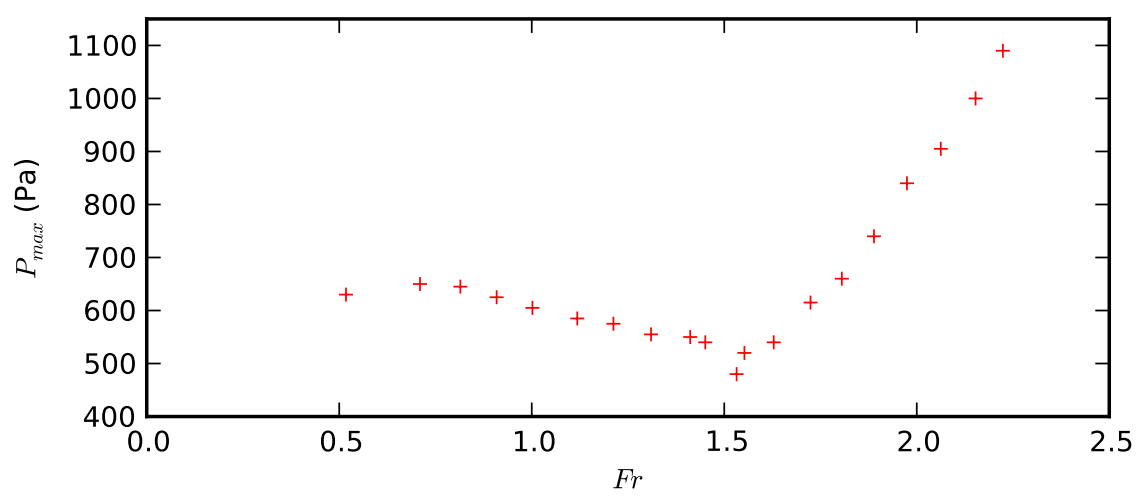

Figure 5: Maximum pressure recorded in the middle of the wall facing upstream.

The pressure does not decrease continuously when $F r$ decreases, but reaches a local minimum at $\mathrm{Fr}=1.5$.

To better understand the interaction of the flow with the structure, we can separate the pressure into two components and study their evolution as the Froude number varies. The theoretical kinetic and gravitational components of the pressure inside the fluid are defined as follows:

$$
\begin{aligned}
P_{k i n}(z) & =\frac{1}{2} \rho u^{2}(z), \\
P_{g}(z) & =\rho g(h-z) \cos \theta,
\end{aligned}
$$

where $u(z)$ is the local flow velocity. 

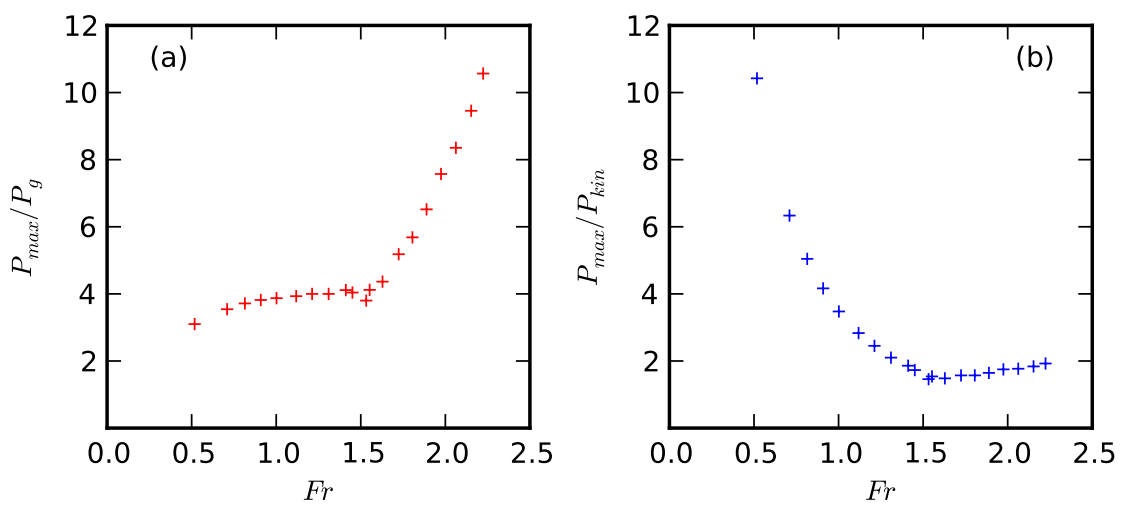

Figure 6: Ratio of the maximum total pressure over the gravitational component (a) and the kinetic component (b).

The kinetic component of the force exerted by the fluid is then:

$$
\begin{aligned}
F_{\text {kin }} & =\int_{0}^{h} \frac{1}{2} \rho u^{2}(z) d z \\
& =\frac{1}{2} \rho h \overline{u^{2}}=\frac{1}{2} \beta \rho h \bar{u}^{2},
\end{aligned}
$$

where $\bar{u}$ is the average velocity of the flow, and $\beta=\overline{u^{2}} / \bar{u}^{2}=1.11$ in our case.

Likewise, the gravitational component of the force is:

$$
\begin{aligned}
F_{g} & =\int_{0}^{h} \rho g(h-z) \cos \theta d z \\
& =\rho g \cos \theta\left(h^{2}-\frac{H^{2}}{2}\right)=\frac{1}{2} \rho g h^{2} \cos \theta .
\end{aligned}
$$

The average pressure components exerted by the fluid are thus:

$$
\begin{gathered}
P_{k i n}=\frac{F_{k i n}}{h}=\frac{1}{2} \beta \rho \bar{u}^{2}, \\
P_{g}=\frac{F_{g}}{h}=\rho g h \cos \theta,
\end{gathered}
$$

The pressure exerted by the flow on the obstacle can be analyzed by calculating, for each slope angle, the ratio between the maximum value of the computed total pressure $P_{\max }$ and the theoretical components $P_{k i n}$ and $P_{g}$.

Figure 6 shows these ratios as a function of $F r$. The ratio $P_{\max } / P_{g}$ is nearly constant for $F r<1.5$ and then grows rapidly for larger $F r$, whereas $P_{\max } / P_{k i n}$ decreases as $F r$ increases, to reach a nearly constant value for $F r>1.5$. 


\section{Summary of the results}

Our simulations have highlighted the existence of a transition between a dead-zone regime and a jet regime, occurring in our case for a value of the Froude number $F r=1.5$. These flow regimes are similar to those observed experimentally by Tiberghien et al. [12] and the value of $F r$ at which the transition occurs in those experiments is close to ours.

The transition between the two regimes is evidenced by the behaviour of the pressure exerted on the obstacle, as figure 3 shows. The first regime corresponds to a slow, smooth rise to the maximum pressure. In the second one however, the pressure peaks quickly then stabilises at a lower value.

The transition is also visible in the evolution of $L_{Z I} / H$ and $P_{\max }$ as $F r$ varies, as can be seen on figures 4 and 5. The maximum pressure exerted on the obstacle does not decrease continuously as $F r$ decreases: its reaches a local minimum in the region of transition.

Finally, tracing the ratios of $P_{\max } / P_{g}$ and $P_{\max } / P_{k i n}$ indicates that the maximum pressure is approximately proportional to its gravitational component in the dead-zone regime, and to its kinetic component in the jet regime. In other terms, for $\mathrm{Fr}<1.5$, the pressure is mostly determined by the weight of the fluid located upstream of the obstacle, while for $F r>1.5$, the velocity of the flow is the relevant factor.

It is interesting to note that these results are similar to observations made on granular flows by Faug et al. [13].

\section{Conclusion}

We have presented a SPH model capable of simulating the interaction of unsteady free-surface flows of a Herschel-Bulkley with a rigid obstacle.

We have observed a transition at $F r=1.5$ between two distinct flow regimes: a dead-zone regime and a jet regime.

In the dead-zone regime, the pressure on the obstacle rises smoothly and its maximum value is determined by its gravitational component. A large dead zone forms, that can have a long-distance influence.

In the jet regime, the pressure peaks quickly after the impact on the obstacle and its maximum value is determined by its kinetic component. In this regime, the extent of the dead zone is very small.

Numerical pressure measurements show that even for small values of $F r$, the force on the obstacle can be significant. As such, it should not be neglected when designing defense structures.

\section{References}

[1] Coussot, P., Steady, laminar, flow of concentrated mud suspensions in open channel. Journal of Hydraulic Research, 32(4), pp. 535-559, 1994. 
[2] Ferrari, A., Dumbser, M., Toro, E. \& Armanini, A., A new 3D parallel SPH scheme for free surface flows. Computers \& Fluids, 38(6), pp. 1203-1217, 2009.

[3] Caccamo, P., Faug, T., Bellot, H. \& Naaim-Bouvet, F., Experiments on a dry granular avalanche impacting an obstacle: dead zone, granular jump and induced forces. WIT Transactions on The Built Environment, 15, pp. 53-62, 2011.

[4] Iverson, R.M., The physics of debris flows. Reviews of Geophysics, 35(3), pp. 245-296, 1997.

[5] Gingold, R. \& Monaghan, J., Smoothed particle hydrodynamics-theory and application to non-spherical stars. Monthly Notices of the Royal Astronomical Society, 181, pp. 375-389, 1977.

[6] Lucy, L., A numerical approach to the testing of the fission hypothesis. The Astronomical Journal, 82, pp. 1013-1024, 1977.

[7] Monaghan, J.J., Smoothed particle hydrodynamics. Annual review of astronomy and astrophysics, 30, pp. 543-574, 1992.

[8] Rodriguez-Paz, M. \& Bonet, J., A corrected smooth particle hydrodynamics method for the simulation of debris flows. Numerical Methods for Partial Differential Equations, 20(1), pp. 140-163, 2004.

[9] Laigle, D., Lachamp, P. \& Naaim, M., SPH-based numerical investigation of mudflow and other complex fluid flow interactions with structures. Computational Geosciences, 11(4), pp. 297-306, 2007.

[10] Morris, J., Fox, P. \& Zhu, Y., Modeling low Reynolds number incompressible flows using SPH. Journal of Computational Physics, 136(1), pp. 214-226, 1997.

[11] Chen, C.L., Generalized viscoplastic modeling of debris flow. Journal of Hydraulic Engineering, 114(3), pp. 237-258, 1988.

[12] Tiberghien, D., Laigle, D., Naaim, M., Thibert, E. \& Ousset, F., Experimental investigations of interaction between mudflow and an obstacle. International Conference on Debris-Flow Hazards Mitigation: Mechanics, Prediction, and Assessment, Proceedings, eds. C. Chen \& J.J. Major, pp. 281-292, 2007.

[13] Faug, T., Beguin, R. \& Chanut, B., Mean steady granular force on a wall overflowed by free-surface gravity-driven dense flows. Physical Review E, 80(2), p. 021305, 2009. 